\title{
EL ACOMPAÑAMIENTO ESPIRITUAL EN CUIDADOS PALIATIVOS
}

\section{SPIRITUAL SUPPORT IN PALLIATIVE CARE}

\author{
Enric Benito', Mónica Dones² y Javier Barbero² \\ ' Consultor senior en cuidados paliativos. \\ 2 Unidad de Cuidados Paliativos, Fundación Instituto San José, Orden Hospitalaria San Juan de Dios, Madrid \\ ${ }^{3}$ Servicio de Hematología, Hospital Universitario La Paz, Madrid
}

\section{Resumen}

Los profesionales sanitarios y especialmente los que trabajamos en oncología y cuidados paliativos somos frecuentemente interpelados por el sufrimiento de nuestros pacientes. El modelo biomédico, es insuficiente para entenderlo y atenderlo. Para ello se requiere ampliar la perspectiva, hacia una visión más abierta que incluya nuestra dimensión espiritual.

La espiritualidad es un universal humano, y se expresa como el dinamismo que impulsa nuestro anhelo inagotable de plenitud y felicidad, que caracteriza la condición humana y que se expresa en la red de relaciones que cada persona establece.

La enfermedad grave y la proximidad de la muerte son oportunidades de apertura a esta dimensión y de sanación. El profesional que conoce este proceso puede acercarse desde la hospitalidad, presencia y compasión y acompañar este itinerario.

En este articulo intentamos explorar de forma resumida qué entendemos por espiritualidad, espiritualidad en clínica, necesidades y recursos espirituales, el itinerario de la persona que atraviesa un proceso de sufrimiento, perdida y/o muerte y las actitudes y herramientas de los profesionales para explorar, atender y acompañar en este espacio difícil y hasta ahora poco transitado.

Palabras clave: Espiritualidad, cuidados paliativos, compasión, presencia, hospitalidad, proceso de morir.

\section{Abstract}

Healthcare professionals, and specially the ones in oncology and palliative care, are frequently questioned about our patients' suffering. The biomedical model is not enough for understanding and caring for it. Therefore, the perspective needs to be widened through a more open vision that includes our spiritual dimension.

Spirituality is a human universal, that expresses as the dynamism that boosts our infinite desire of plenitude and happiness, that characterizes human condition and that is expressed in the net of relationships stablished by each person.

Severe illness and proximity to death are opportunities of an opening to this dimension and of healing. Professionals that know this process can approach from hospitality, presence and compassion, and accompany patients in this journey.

In this paper, we try to explore in a synthesized way, what is understood by spirituality, clinical spirituality, spiritual resources and needs, the journey of a person going through a suffering, loose or dying process, and the attitudes and tools of professionals to explore and accompany in this difficult and not very busy path.

Keywords: Spirituality, palliative care, compassion, presence, hospitality, dying process, end of life care.

\section{Correspondencia:}




\section{EL ACOMPAÑAMIENTO ESPIRITUAL EN CUIDADOS PALIATIVOS}

"Un encuentro en profundidad con el que está muriendo puede llevarnos a un auténtico despertar, a una profunda transformación de nuestra forma de entender y vivir la vida."

Sogyal Rinpoché

\section{PLANTEAMIENTO DEL PROBLEMA}

Los profesionales sanitarios y más específicamente los que trabajamos en oncología y cuidados paliativos somos frecuentemente interpelados por el sufrimiento de nuestros pacientes. Éste puede ser aliviado ocasionalmente con intervenciones específicas, tratando un dolor o el insomnio o ayudando a adaptarse a las pérdidas.... En otras ocasiones nos enfrentamos no a un problema, sino al misterio. A las preguntas existenciales, ¿por qué a mí?, ¿por qué debo morir?, ¿qué me espera?, no tenemos respuestas contundentes. Aquí no podemos resolver ni solucionar $y$, a veces, la tentación es abandonar, huir, argumentando que esta parte más dura ya no es competencia nuestra. O simplemente no sabemos qué hacer y evitamos entrar en un territorio para el que no tenemos mapas y tememos perdernos o sencillamente hacer daño. En este articulo intentaremos explorar de forma resumida qué entendemos por espiritualidad, espiritualidad en clínica, necesidades y recursos espirituales, el itinerario de la persona que atraviesa un proceso de sufrimiento, perdida y/o muerte y las actitudes y herramientas de los profesionales para explorar, atender y acompañar en este espacio difícil y hasta ahora poco transitado. Vamos a empezar comentando un caso clínico.

\section{EL CASO DE PABLO}

Por su condición de oncólogo dedicado a los cuidados paliativos, uno de los auto- res (EB) fue consultado por un caso difícil, que estaba siendo atendido por otros compañeros oncólogos en una clínica privada. Se trataba de Pablo, un joven de 24 años, que hubo de suspender su último curso de biología en la universidad, tras el diagnóstico de meduloblastoma. El tumor recidivado progresaba tras haber agotado todos los tratamientos (cirugía, quimio y radioterapia). Lo que me llamó la atención al entrar en la habitación de la clínica donde Pablo estaba ingresado, fue el ambiente de derrota del entorno. En pleno día, las ventanas cerradas ... solo la luz indirecta, un joven alopécico con signos de Síndrome de Cushing, encamado y enganchado a un suero. Allí estaban -como más tarde supe- la novia y algunos amigos, sentados en la cama y una madre con niveles de ansiedad que inundaban la habitación. Tras explorarlo y hablar con él y la madre, comprobé que tenía cefaleas incontroladas, somnolencia, astenia, impotencia funcional por inestabilidad de origen cerebeloso y una tristeza oceánica. La familia estaba muy asustada ya que el médico que pasaba cada dos o tres días, se lamentaba de que no podía hacer nada más y que iya llevaba mucha morfina! Ellos no veían salida a la situación. Tras comprobar con los oncólogos que lo trataban, la ausencia de posibilidades de seguir más tratamiento del que llaman "activo", acordamos con ellos y la familia el traslado de Pablo a nuestra unidad de Cuidados Paliativos donde pensábamos quedaban muchas cosas por hacer, probablemente las mas importantes. Allí hicimos una valoración integral y una lista de problemas, físicos (control del dolor y el cuadro de hipertensión endocraneal, inmovilidad, etc.), emocionales (explorar la información pronostica e ir facilitando la adaptación a la situación, intervención con la familia para promover la aceptación del proceso), sociales (acabar con el aislamiento, trabajar el entorno de negación, ir ayudando a los padres y familiares a aceptar lo que nos venía etc.) y espirituales (explorar 
y acompañar las necesidades y los recursos de Pablo y de la familia, y compartir nuestra experiencia de que esta etapa puede ser una de las mas intensas y ricas de la vida de todos ellos).

En menos de 48 horas del traslado al entorno acogedor, luminoso y animado de nuestra unidad habíamos conseguido un buen control del dolor, movilizaciones en silla de ruedas con salidas a la terraza de la habitación y, después, al jardín, un cambio de ánimo tanto de él como de la familia, que empezó a sentir que se la hacían cosas y que Pablo estaba mejor. A los pocos días un Pablo mas abierto, confiado y esperanzado y que nunca había tenido información pronostica clara, nos abordó con la pregunta del millón: "¿Cuándo me van a poner el tratamiento?" Nos pusimos a la altura de sus ojos y, conmovidos por su confianza y vulnerabilidad, le dijimos que ya le estábamos tratando, pero insistió preguntando por la nueva quimioterapia. Le dijimos que no le podíamos poner más ya que iba a ser más tóxica que eficaz... entonces, más preocupado dijo:

- Cirugía ya me han dicho que no me pueden hacer iy la radioterapia?

- Ya se han puesto las dosis máximas tolerables por tu cerebro, ahora te haría daño, no vamos a tratar más el tumor, éste ya no puede ser nuestro objetivo, desafortunadamente. Ahora vamos a tratarte a ti y a cuidar de ti.

Hubo un silencio en el que nos mantuvimos conectados con él y al poco tiempo, mientras iba tomando conciencia de lo que ocurría, preguntó bastante asustado:

- ¿Entonces, me voy a morir?

Sus ojos estaban pidiendo ayuda y, desde el vínculo de acogida y con la máxima serenidad y ternura posible le dijimos:

- Pablo, ¿no lo habías pensado nunca?

Aquí hubo una espacio largo en que fue confirmando sus intuiciones $y$, conmovido, se puso a llorar. Estuvimos largo rato con él, después explicamos la conversación a sus padres.

Pasó los dos días siguientes Ilamando a los amigos y despidiéndose de todos. Hasta que, en un momento dado, tomamos de nuevo la iniciativa para decirle:

- Pablo es cierto que no te podemos curar y en algún tiempo esto irá peor, pero de momento debes decidir si te quedas esperando la muerte, desde la tristeza y el miedo o prefieres vivir este tiempo que te pertenece con los tuyos de la manera que tú mismo elijas.

A partir de este momento empezó un cambio. La habitación y sobre todo la terraza de su habitación, donde sus amigos de la facultad habían traído una barbacoa, se convirtió en una de las zonas más activas y festivas del hospital. Los fines de semana salía con sus padres y familia, que compartían la idea de vivir intensamente lo que les quedaba, iban a la casa de campo de los tíos y algunas tardes, su novia traía de la cuadras próximas al Hospital el caballo que su padre le había regalado a los 20 años y que, como buen menorquín, había adiestrado él mismo. Pablo ya no podía montar pero lo acariciaba y disfrutaba de ver a su novia hacerlo. Pasaron casi 3 meses desde aquella conversación tan difícil. Se había convertido en parte de nuestra historia, las auxiliares y las enfermeras que le cuidaban y los médicos nos habíamos implicado probablemente mas allá de lo puramente profesional. Y al mismo tiempo que nos implicábamos, nos hacíamos conscientes de que la partida de Pablo nos iba a doler mucho.

Pablo empezó a perder visión, y equilibrio, se pasaba el día encamado, al principio escuchando música y recibiendo vistas y, poco a poco, se fue encerrando en sí mismo y me volvió a preguntar.

- Esto ya está cerca, ¿Cómo será?. ¿Cómo sabré si lo hago bien?, ¿Tú me guiarás?. 
- Sí Pablo, vemos que ya estás cada día mas cansado y si, hemos visto muchas veces cómo va esto y aunque el camino lo vas a hacer tú solo, nosotros conocemos el mapa, sabemos lo que ocurre, te guiaremos y verás cómo todo está bien organizado. Cuando llegue el momento nosotros estaremos contigo para que puedas encontrar la forma de soltar, aflojar, dejarte y comprobar que algo muy sólido y muy tierno a la vez te sostiene.

Evidentemente tuvimos que trabajar con la novia, los amigos, los padres y a todos irles pidiendo que dejaran partir a Pablo, que le dieran permiso para irse, ya que se estaba acercando la hora y que todo iría con el menor sufrimiento posible.

Los que empezábamos a estar inquietos éramos los profesionales, pocas veces habíamos entrado en tanta intimidad con un paciente y su familia y, sobre todo, durante tanto tiempo.

Unos 8 días antes de que falleciera, barruntando lo que se nos venia encima decidimos hacer una sesión de equipo de las que Ilamamos "Grupo de apoyo". Es una dinámica sencilla tomada de los grupos de duelo o terapias de apoyo grupal. Hay unas normas de confidencialidad y de respeto al tiempo de cada uno, que recuerda el moderador si hace falta y en el tiempo de cada uno se expresa lo que está pasando y cómo uno se siente o calla mientras los demás sin intervenir acogen como caja de resonancia lo que el que expresa pone en el centro. Los demás practican escucha activa sin intervenir y el que se expresa tiene permiso para lo que considere: Ilorar, lamentar, blasfemar... Menos agredir...todo está permitido. Convocamos la reunión explícitamente para hablar del caso de Pablo a una hora que pudieran encontrarse los turnos de mañana tarde y que pudieran venir las personas del turno de noche que quisieran. Fue una sesión muy intensa. Acudimos muchos. Algunos Iloramos, expresamos la aparente injusti- cia de la muerte de una persona y en una familia tan jóvenes y tan buenos, compartimos nuestra impotencia por no poder hacer más y nuestra satisfacción por haber podido conocerles y acompañarles. Fue higiénico para nosotros poder - metafóricamente- encender una hoguera en el centro de la reunión donde ardieron nuestra frustración, nuestra tristeza y nuestro apego a un paciente que nos había seducido con su sencillez y bondad.

Pablo, poco a poco, se fue confiando, relajando y abandonando. Acompañarle era fácil y gratificante, no había síntomas molestos a excepción de la astenia y somnolencia progresivas, no hubo resistencia ni miedo. Se habían estado preparando durante meses, cada cena, cada noche de guitarras podía ser la ultima y Pablo sus padres y sus amigos se habían ido forjando la idea de una despedida triste pero plena, habiendo vivido conscientemente lo que se acercaba y con risas, música, y cenas en la terraza. Ahora había Ilegado la hora de soltar, dejarse y entrar en paz a lo que venía. Los últimos días nos comentó a su madre y alguno de los más cercanos que sabía que iba a reunirse con su abuelo, la persona que tanto había querido y que sabía que le estaba esperando, aquello le daba serenidad y confianza.

Han pasado años de esta historia. La madre de Pablo, una vez cerrado el duelo, dedicó algunos años posteriores a trabajar en la asociación de Voluntarios de cuidados paliativos y sentimos que haber acompañado a cerrar esta biografía nos había transformado a muchos.

Como en todas las historias de acompañamiento siempre hay tres actores. Un protagonista: el que se va; y unos coprotagonistas, los familiares que intentan aceptar, apoyar y dejar partir y, en tercer lugar, unos profesionales que además de cuidar y acompañar deben saber cuidarse, tratar de no sobreimplicarse $y$, si lo hacen, saber elaborar -si es posible juntos- el reconoci- 
miento de la realidad y aceptar que somos vulnerables, frágiles y recordar que el trabajo bien hecho no es resolver ni curar, sino acompañar desde la presencia y la compasión.

Las lecciones que aprendemos acompañando nos pueden abrir a una nueva perspectiva de nosotros mismos y de la vida, haciéndonos cambiar las prioridades y madurar como personas $y$, en la medida que podamos ir aceptando nuestra propia vulnerabilidad e incluso nuestra propia muerte, abrirnos con mayor intensidad al gozo de la vida.

\section{INTRODUCCIÓN, CONCEPTOS Y DEFINICIONES. LA ESPIRITUALIDAD COMO UNIVERSAL HUMANO}

Aunque atender el sufrimiento de nuestros pacientes forma parte de la esencia de los cuidados paliativos ${ }^{(1)}$, en la práctica clínica observamos dificultades para acercarnos a la persona que sufre y para reconocer, atender y acompañar este proceso.

Nos cuesta diagnosticar y atender el sufrimiento, en parte por falta de un marco académico que nos permita una aproximación estructurada. También lo obviamos en la medida en que pertenece al ámbito de lo subjetivo, mientras nuestro modelo biomédico y nuestra formación apenas contemplan la subjetividad como parte de la realidad clínica ${ }^{(2)}$.

En las dos últimas décadas hemos asistido a un incremento exponencial de publicaciones sobre la atención espiritual en clínica, con diferentes propuestas para explorar y atender las necesidades y acompañar los recursos de los pacientes.

Hace años Cicely Saunders ${ }^{(3,4)}$ puso énfasis en la necesidad de abordar el "dolor total", reconociendo los aspectos psicológicos, sociales y espirituales del sufrimiento. Hoy la mayoría de profesionales de cuidados paliativos son todavía más expertos en el control de síntomas y ma- nejo de fármacos, que en la desesperanza, en propiciar la reconciliación con la propia biografía, en promover la búsqueda de sentido o en facilitar la aceptación de la muerte. Estos suelen ser escasamente atendidos, por considerarlos alejados de nuestra competencia o por falta de recursos personales para abordarlos. Entre tanto diariamente somos interpelados por el insomnio, la ansiedad y por el sufrimiento de nuestros pacientes.

La persona con una enfermedad terminal suele intuir la proximidad de la muerte y el sufrimiento es la amenaza que se cierne sobre todo lo conseguido, que ahora parece que va a deshacerse. Para muchas personas, especialmente si no se han preparado para ello, esta etapa puede ser vivida como un asalto a la propia construcción vital -todo aquello con lo que nos hemos identificado- y que ahora, se intuye que acabará por desmoronarse.

La NANDA (North American Nurses Diagnosis Association) hace referencia al Sufrimiento Espiritual dentro de sus diagnósticos desde 1978. Lo define como: "Deterioro de la capacidad para experimentar e integrar el significado y propósito de la vida mediante la conexión con el yo, los otros, el arte, la música, la literatura, la naturaleza y/o un poder superior al propio yo". En su diagnóstico Disposición para mejorar el bienestar espiritual (00068), hace referencia a la espiritualidad como la integración del significado y propósito de la vida ${ }^{(5)}$.

Aquí, desde la experiencia clínica y el estudio, tanto de la bibliografía como de las tradiciones de sabiduría, proponemos un reencuentro con lo que siempre ha sido nuestra naturaleza esencial como seres humanos, nuestra dimensión espiritual. Somos seres de naturaleza espiritual, seamos o no conscientes de ello. La Vida nos sostiene, nos nutre y nos conecta con nosotros, con los demás y con la naturaleza y el universo. Esta Vida que fluye en 
nosotros aspira a su máximo desarrollo y expresión en cada uno, anhelando el progresivo despertar de nuestra conciencia.

La espiritualidad es un universal humano, expresión de ese dinamismo que impulsa nuestro anhelo inagotable de plenitud y felicidad, que caracteriza la condición humana y que se expresa en la red de relaciones que cada persona establece.

Esta naturaleza espiritual, profunda, íntima e intangible que nos caracteriza y confiere nuestra humanidad, es el dinamismo que pertenece y se experimenta a un nivel transracional, transpersonal y transconfesional. Es decir se descubre y se vive en, pero sobre todo más allá de lo cognitivo, de lo individual y de lo creencial, trascendiéndolo e integrándolo.

Las tradiciones de sabiduría y las religiones han reconocido esta dimensión humana con diferentes nombres: el reino de los cielos en nuestro interior del evangelio, el Cristo que vive en mí de S. Pablo, el espíritu en el alma del Maestro Eckart, la conciencia sustrato del budismo, el secreto del islam, la apertura original del taoísmo, la kundalini del yoga tántrico, el Atman en el hinduismo, el pedestal interior de Marco Aurelio, el Daimon de Sócrates etc.

Las tradiciones de sabiduría y las religiones, han elaborado marcos conceptuales, ritos y preceptos y han abierto caminos para acceder a la experiencia espiritual. Todas indican el camino hacia la experiencia, aunque ninguna puede apropiarse con exclusividad de esta realidad que inspira y trasciende toda creencia.

Las personas gozamos de distintos grados de madurez espiritual, al igual que los humanos alcanzamos diferentes grados de desarrollo moral(6,7). Según Wilber ${ }^{(8)}$ estas etapas son creencia, fe, experiencia directa y adaptación permanente. El grado de conciencia espiritual no modifica el valor ni la dignidad de cada persona, tan solo indica el grado de apertura de cada uno a la propia esencia en un momento dado.
Existe un itinerario personal para descubrir la propia espiritualidad. El ser humano es un proyecto en construcción y en la biografía de cada uno hay periodos especialmente fructíferos en el despertar espiritual, experiencias cumbre ${ }^{(9,10)}$, frecuentemente asociados a situaciones de crisis, en los que la propia cosmovisión se desmorona y la persona, en medio del dolor de la pérdida, puede revisar y reconstruir a un nivel superior la percepción que tenía de la realidad y de sí mismo.

Todos vivimos momentos de crisis/ oportunidad: La enfermedad, el sufrimiento y la proximidad de la muerte son ocasiones especiales para la emergencia espiritual, en ellas se abre la oportunidad de encuentro consigo mismo, con los demás y con lo que nos trasciende y nos permite experimentar lo que somos en nuestra profundidad.

\section{LA ESPIRITUALIDAD EN CUIDADOS PALIATIVOS}

El reciente interés en dotarnos de un marco conceptual ha generado definiciones de espiritualidad en el entorno clínico. La conferencia de consenso(11) de 2008 define: "La espiritualidad es el aspecto de la humanidad que relaciona la manera en que los individuos buscan y expresan un significado y propósito, y la manera en que experimentan su conexión con el momento, consigo mismos, con los demás, con la naturaleza y con lo significante o sagrado."

Según el Spiritual Care Task Force de la EAPC en 2011 (12): "La espiritualidad es la dimensión dinámica de la vida humana que relaciona la forma en que las personas (individuos o comunidades) experimentan, expresan y/o buscan un significado, propósito y transcendencia, con la forma en que conectan con el momento, consigo mismos, con los demás, con la naturaleza, con lo significante y/o con lo sagrado." 
Según la conferencia de expertos de Ginebra de 2013 ${ }^{(13)}$ : "La espiritualidad es un aspecto dinámico e intrínseco de la humanidad a través del cual las personas buscan un significado, un propósito y una transcendencia últimas y experimentan una relación consigo mismos, con la fa- milia, con los demás, con la comunidad, con la sociedad, con la naturaleza, con lo significativo y con lo sagrado. La espiritualidad se expresa a través de las creencias, los valores, las tradiciones y las prácticas". La tabla 1 recoge un resumen de la Conferencia de 2008.

\section{Tabla 1. El acompañamiento espiritual como dimensión de los cuidados paliativos: Informe de una conferencia de consenso(11)}

- Los modelos de cuidados espirituales ofrecen un marco de referencia para que los profesionales de la sanidad puedan conectar con sus pacientes, escuchar sus miedos, sueños y dolores, colaborar con sus pacientes como aliados en su cuidado y proveer, a través de la relación terapéutica, una oportunidad de sanación.

- En este contexto, la sanación se distingue de la curación. Se refiere a la habilidad de una persona para encontrar solaz, consuelo, conexión, significado y propósito en medio del sufrimiento, la desazón y el dolor.

- Los cuidados espirituales están cimentados en marcos teóricos importantes, uno de los cuales es el Modelo Bio-psicosocial-Espiritual de Cuidados

- Otro de los fundamentos es un modelo de cuidados centrado en el paciente, en el que el enfoque de cuidados se centra en éste y en su experiencia de la enfermedad, en vez de centrarse en exclusivamente en la enfermedad.

- La visión de la persona como ser-en-relación es piedra angular del acompañamiento.

- La vida es esencialmente relaciones; y relación como tal implica trascendencia, un ir más allá de sí mismo por parte de aquello involucrado en la relación.

- La enfermedad puede ser comprendida como una disrupción en las relaciones adecuadas que constituyen la unidad e integridad de lo que conocemos como ser humano.

- La profesión sanitaria es, intrínsecamente, una profesión espiritual. Cuando interactúan un profesional de la salud y un paciente en una relación profesional, la transformación que tiene lugar es inherente al modelo propuesto de cuidados espirituales.

- Al cuidar de las personas que sufren, para el profesional de la salud se abre la oportunidad de transformación personal. Para estar abierto a ella, el profesional debe tener conciencia de las dimensiones espirituales en su propia vida y apoyarse en un proceso de reflexión para la práctica de la presencia compasiva con los pacientes.

- Cuando los profesionales de la salud toman conciencia de sus propios valores, creencias y actitudes, especialmente respecto a su propia condición mortal, crean conexiones más profundas y significativas con sus pacientes.

- La espiritualidad entendida como nuestra naturaleza esencial de donde surge nuestro anhelo inagotable de plenitud es un universal humano. Se expresa a través de nuestra red de relaciones, las que establecemos con nosotros mismos, los demás y lo que nos trasciende.

- "Los que sufren no son los cuerpos, son las personas", El sufrimiento se da en el ámbito subjetivo: centrados sólo en lo objetivo los profesionales no lo pueden explorar ni atender.

- Los clínicos nos encontramos sufrimiento evitable y no evitable. Hay un sufrimiento que forma parte de nuestra condición existencial, pertenece a la experiencia del misterio y se puede trascender y acompañar, pero no resolver.

- Disponer de un modelo antropológico, que entiende a la persona como parte de la conciencia que aspira a la integridad, a la integración y la plenitud, puede ayudarnos a entender y atender el sufrimiento.

- El itinerario del proceso de trascendencia del sufrimiento se reconocen tres etapas, identificadas como:

"CAOS, etapa muda, de enclaustramiento o de lucha.

» ACEPTACION, de entrega o etapa expresiva o de transición

" TRASCENDENCIA, maduración o de nueva identidad

- La trascendencia del sufrimiento, y disolución de la perspectiva limitada de la persona, abre un panorama ampliado de sí mismo y de la realidad, y es el camino más poderoso para restaurar la integridad a un nivel de conciencia superior.

- La sanación como proceso de recuperación de la integridad y armonía, a través de trascender el sufrimiento, puede ser una propuesta clave de la intervención compasiva en el acompañamiento espiritual.

- Las herramientas del acompañante son HOSPITALIDAD, PRESENCIA Y COMPASION

- La presencia ecuánime y compasiva del terapeuta posibilita que el que sufre pueda adentrarse y atravesar y trascender conscientemente la visión limitada de si mismo, y entregarse a la profundidad de su Ser.

- El acompañante debe desarrollar un nivel de conciencia, ecuanimidad y compasión y ser consciente de sus limites para poder acompañar. 


\section{PROPUESTA DE UN MODELO PARA NUESTRO ENTORNO CULTURAL. GRUPO DE ESPIRITUALIDAD DE LA SECPAL (GES)}

La SECPAL (Sociedad Española de Cuidados Paliativos) cuenta con un Grupo de Trabajo sobre Espiritualidad en Cuidados Paliativos (GES) formado por clínicos que han propuesto desarrollar una atención integral, que contemple la atención al sufrimiento y a las necesidades y recursos espirituales de los pacientes y sus familiares. Desde 2004 trabaja con una composición multiprofesional, y una perspectiva antropológica integral, humanista, y transconfesional con la misión de:

"Promover entre pacientes, familiares y profesionales la oportunidad de transformar el proceso de morir en una experiencia compartida de crecimiento personal y de toma de conciencia de nuestros recursos y necesidades espirituales".

Desde el GES hemos definido Espiritualidad como: "Nuestra naturaleza esencial de donde surge un anhelo inagotable de plenitud, que concretamos en la búsqueda de sentido, conexión y trascendencia. Es un universal humano que nos caracteriza a todas las personas y que puede estar vivida y/o expresada, o no, a través de la religión"(14).

\section{Modelo de Espiritualidad SECPAL}

Para aproximarnos al territorio hemos construido un mapa, basado en estas asunciones:

a) Nuestra naturaleza esencial como seres humanos es espiritual. Todos los grupos de expertos ${ }^{(10-13)}$ coinciden en la dimensión dinámica de la espiritualidad, así como en conceptualizar la persona, en su dimensión espiritual, como un ser en relación. Hay también acuerdo respecto a un mínimo de tres niveles de estas relaciones: inter-, intra-, y transpersonal ${ }^{(15)}$. Tal y como afirma Balfour Mont(16) "los humanos son intrínsecamente espirituales, puesto que todas las personas están en relación con sí mismos, con los otros, la naturaleza y lo significativo o sagrado".

b) Siguiendo a V. Frankl: "La espiritualidad puede ser un recurso poderoso para trascender el sufrimiento que acompaña la pérdida, así como para promover el desprendimiento y la aceptación implicados en el proceso de morir"/17). El cuidado espiritual está basado en el modelo de cuidado bio-psico-social-espiritual propuesto por Sulmasy ${ }^{(18)}$ y en la perspectiva integral de la persona que nos ofrece Casell(19), mejorando los recursos de los pacientes y facilitando la trascendencia del sufrimiento.

c) El sufrimiento puede verse como una amenaza de pérdida de integridad, coherencia y conciencia de pertenencia, pero también como una llamada a la plenitud. El modelo asume que la muerte es más que un hecho biológico. Es un hecho humano, social y espiritual y también una oportunidad especial que presenta el reto de descubrir y sumergirnos en nuestra naturaleza espiritual y $\operatorname{crecer}^{20}$. En este contexto, el cuidado espiritual debería ser visto como un acercamiento profesional y ético a esta oportunidad que se ofrece para la sanación.

d) La sanación ha sido definida por Mount y Kearney ${ }^{(21,22)}$ como un proceso relacional que implica movimiento hacia la experiencia de integridad y plenitud. Puede ser 
facilitada por las intervenciones de los cuidadores, pero depende de un potencial innato dentro del paciente $^{(23)}$. El cuidado espiritual basado en la conexión espíritu-a-espíritu (entre paciente y cuidador) aspira a facilitar la sanación.

e) La salud necesita ser entendida no sólo como un constructo físico, sino también como un aspecto de nuestra red de relaciones, tal y como vienen sugiriendo muchos autores ${ }^{(24-27)}$.

Este marco conceptual de aproximación a la espiritualidad coincide con la mayoría de publicaciones, así Reed en1992 la definió como "la propensión a dar significado a través de un sentido de relación con las dimensiones que trascienden al ser de tal manera que empodere y no devalúe al individuo. Esta relación puede ser experimentada intrapersonalmente (como una conexión con uno mismo que aspira a la coherencia y el sentido), interpersonalmente (en el contexto de otros y del entorno natural que aspira a la armonía y comunicación) y transpersonalmente, en referencia al sentido de relación con lo desconocido, el misterio, Dios, o un poder más grande que uno mismo"115). Veinte años más tarde, Meezenbroek ${ }^{(27)}$ apunta la necesidad de adoptar un acercamiento no-teístico, en el que la espiritualidad se defina como el anhelo de experimentar la conexión con la esencia de la vida y sus dimensiones de conexión con uno mismo, con otros y la naturaleza y la conexión con lo trascendente.

Partiendo de este modelo antropológico que reconoce la naturaleza espiritual y la persona como ser en relación, el GES construyó y validó un cuestionario basado en los recursos y necesidades de conexión en estos tres niveles (intrapersonal, interpersonal y transpersonal) para explorar el grado de plenitud alcanzado en la biografía de cada paciente, y así identificar aque-
Ilas áreas de necesidades a ser evaluadas y atendidas y los recursos disponibles para ello(28).

El cuestionario $\mathrm{GES}^{(28)}$ es una guía para la evaluación de las tres dimensiones esenciales: los aspectos más significativos de nuestras relaciones con uno mismo (necesidad de sentido y coherencia), con otros (armonía en nuestras relaciones con las personas que más nos preocupan y necesidad de sentirnos amados y amar) y con lo trascendente (necesidad de tener esperanza y dejar un legado que va más allá de nosotros mismos). Las preguntas son tan solo una orientación para abordar explorar y atender aquellos aspectos de las relaciones intra, inter y transpersonales que pueden surgir como recursos o necesidades a validar o acompañar respectivamente. La herramienta de acompañamiento nunca será un cuestionario sino el profesional o la persona que encarnando la sabiduría y la compasión, se acerca desde la humildad, el respeto y la simetría moral, para acompañar el viaje del que se está despidiendo. Y para ello, conviene conocer el siguiente apartado.

\section{Itinerario del proceso de enfermar y} morir: del caos a la trascendencia

Kathleen D. Singh, desde su amplia experiencia en acompañamiento, describió $^{(29)}$ como un gran porcentaje de los pacientes, en el último tramo de su vida, independientemente de que tuvieran o no deseo consciente de trascendencia, parecían entrar en un estado modificado de conciencia que llamó: "experiencia de la cercanía de la muerte" que, entre otros, incluyen sentimientos de conexión y paz. Esta autora recuerda que los pacientes describieron la transformación que se da al final de la vida como: "el descubrimiento individual acelerado de sí mismo (self)".

Singh indica que la experiencia de la proximidad de la muerte puede, por sí 
misma, concentrar en pocos momentos la oportunidad de crecimiento espiritual que puedan dar años de intensa disciplina espiritual.

Shaver ${ }^{(30)}$, propone una nueva definición de sufrimiento en la que expresa el dinamismo del proceso de sanación: "El sufrimiento es un proceso dinámico y transformador a través del cual la naturaleza incompleta y fracturada del self comienza a re-emerger en la conciencia. El disconfort y la ansiedad asociados al sufrimiento ocurren como resultado de esta re-emergencia y también del intenso y progresivo anhelo de las partes no integradas o asimiladas del self. El sufrimiento puede ser conscientemente transcendido a través de intervenciones específicas orientadas a promover la unidad del self. Éstas incluyen la atención consciente, reflejar y validar, así como el amor incondicional. La resolución positiva del sufrimiento puede Ilevar hacia una unificación reforzada del self, con un amplio cortejo de emociones, autoestima y hacia una naturaleza trascendente. El sufrimiento supone un desencadenante importante en el despliegue de la conciencia individual". Singh ${ }^{(29),}$ partiendo de la observación del proceso que frecuentemente siguen los enfermos en los últimos días, propone un modelo para explicar la adaptación psicoemocional al proceso y los pasos que se dan en esta etapa se exponen en la figura 1.
Una etapa de caos, que engloba las fases descritas por Kübler Ross ${ }^{(31)}$ caracterizada por la lucha, la negación, la depresión, negociación, y por el miedo a perder lo que consideramos nuestra integridad (es el miedo a la pérdida del EGO) o el miedo a la pérdida de los mecanismos compensatorios descritos por Shaver ${ }^{(30)}$. Posteriormente, el enfermo llega a una fase que llama surrender o rendición, que podríamos traducir como entrega, aceptación, soltar, dejarse, dejar de luchar, etc., lo que en la práctica coloquialmente decimos" tirar la toalla", pero no como un planteamiento de derrota, sino de entrega. Finalmente, cuando se ha hecho esta entrega, la persona entra en un nuevo espacio de conciencia que Singh Ilama trascendencia, un estado modificado de conciencia, un espacio de paz, serenidad, gozo, bienestar, que podemos observar en las fases finales de algunos enfermos.

Esta misma autora describe el proceso diciendo: "El único camino hacia el ámbito transpersonal es a través del examen y entrega completa del sentido personal del yo. No hay otra puerta de entrada. Solamente hay la puerta de la verdad del sufrimiento. Nuestra creencia de ser un ser separado de la realidad es el origen de nuestro sufrimiento. Cuando en una enfermedad terminal uno comprende que no tiene escapatoria de su sufrimiento, entonces empieza a compren-

\section{Figura 1. modelo para explicar la adaptación psico-emocional al proceso de final de vida}

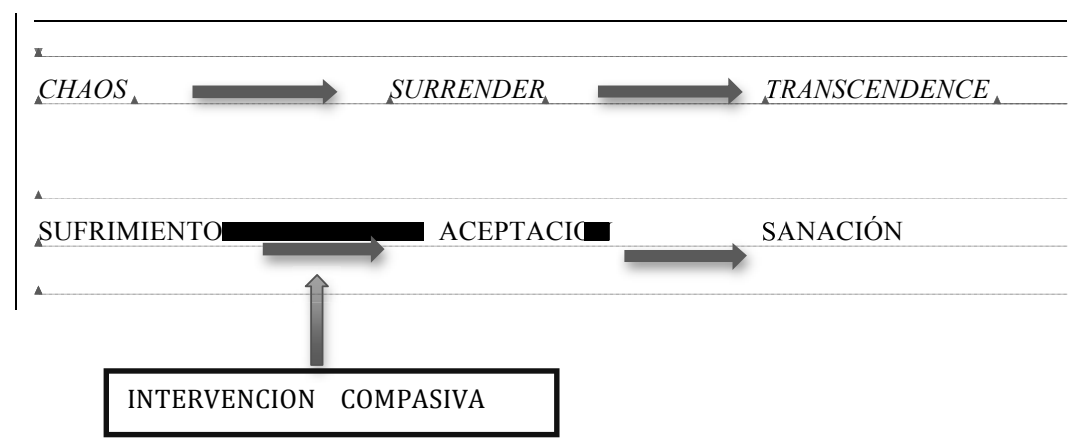


der. Las intuiciones que abren nuestra nueva comprensión solo llegan a través de los agujeros que se producen cuando se agota nuestra resistencia, entonces empezamos a percibir la verdadera naturaleza de nuestro SER, entonces empezamos a disolver nuestra mente ordinaria.

Lo que ocurre al ir disolviendo nuestra mente ordinaria es que la Luz empieza a entrar a través de las brechas de su ausencia. Este es el principio de la plenitud de conciencia, es el principio de la apertura espiritual. Es la entrega de la lucha, del apego, del desespero".

No se trata de una cuestión de renunciar al ego, es una cuestión de explorarlo manteniendo sus competencias y eliminando su exclusividad. En el cristianismo se describe como experimentar y aceptar la presencia y cercanía de Dios: "Padre hágase tu voluntad y no la mía".

Este es un modelo muy interesante ya que, aunque solo sea tomado como una hipótesis de trabajo, es un mapa que nos guía hacia dónde debemos orientar la búsqueda interior de los enfermos, cómo facilitar el proceso.

El que no sabe a dónde va no puede acompañar a nadie y nosotros como profesionales necesitamos referencias de cómo es el proceso, mapas del territorio por el que transcurren los últimos días de nuestros pacientes, para poder acompañar, orientar o sugerirles determinados cami- nos, sus obstáculos y posibles salidas. Este modelo es una propuesta que nos puede ser útil para su acompañamiento.

\section{El acompañamiento espiritual como intervención terapéutica}

Este supone una intervención compasiva hecha desde el corazón, en sintonía con el sufrimiento del enfermo, donde el profesional comparte al mismo tiempo su vulnerabilidad, y su propia experiencia trascendente y aporta su presencia como sanador herido. Esta intervención puede ayudar al enfermo a cruzar el puente que lleva del sufrimiento a la aceptación, la entrega y hacia la trascendencia y con ello a la sanación. Entendida ésta como recuperación de la integridad y coherencia a un nivel de conciencia superior al previo.

A través de la intervención compasiva, el que sufre y el que la acompaña acaban en un espacio distinto caracterizado por el crecimiento y la visión más madura de la realidad.

Es crucial para atender el sufrimiento, la disponibilidad del clínico para diagnosticarlo, su interés y disponibilidad para conocer sus causas, identificar el grado y los recursos, estrategias y posibilidades del enfermo para afrontarlo.

Es decir que, de forma esquemática, los pasos para la intervención se exponen en la tabla 2.

\section{Tabla 2. Intervención Compasiva}

Intervención Compasiva

$\checkmark \quad$ Establecer una relación de confianza y un vínculo terapéutico

$\checkmark$ Identificar el sufrimiento

$\checkmark$ Identificar las causas del sufrimiento

$\checkmark \quad$ Intentar resolver o desactivar las amenazas que puedan resolverse

$\checkmark$ Explorar los recursos y capacidades del paciente para trascender su sufrimiento

$\checkmark$ Intervención Compasiva, orientando al paciente hacia la búsqueda de una visión transcendida de lo que percibe como amenaza. 
Las herramientas del clínico que acompaña: hospitalidad, presencia y compasión

Nuestro modelo propone una intervención en el sufrimiento y la herramienta por excelencia es la propia persona del profesional, que dispone de unas actitudes que le pueden ayudar a facilitar el paso hacia la aceptación. A estas actitudes les hemos Ilamado hospitalidad, presencia y compasión.

Henri Nowen ${ }^{(32)}$ nos dejó el paradigma del "sanador herido" que describe muy bien cómo el acompañamiento espiritual parte de compartir la misma condición frágil y vulnerable. Viene a decir que para acompañar hay que crear un espacio donde el otro pueda sentirse acogido, a esto lo Ilama hospitalidad como respuesta básica a la condición humana de soledad.

Dice: "La hospitalidad es la virtud que nos permite romper la estrechez de nuestros miedos y abrir nuestras casas al extraño, con la intuición de que la salvación nos llega en forma de un viajero cansado" [...] "¿Qué exige la hospitalidad para convertirse en poder curativo? En primer lugar que el que hospeda se sienta en su propia casa" (es decir que se encuentre bien consigo mismo, sin miedo, en paz) " $y$, en segundo lugar, que cree un lugar libre y sin miedo para el visitante inesperado ${ }^{\prime \prime 32}$ (un espacio de serenidad y confianza donde el otro pueda sanar, una posada donde reponerse de las heridas).

La búsqueda debe hacerse profundizando en el sufrimiento, atravesándolo, no rechazándolo. Dicen los toreros que la forma de superar el miedo al toro es mirarlo a los ojos. En el momento que miras el miedo a los ojos, desde la conciencia plena, puedes tomar conciencia de cual es su verdadero poder y desactivar el miedo. En el momento en que el paciente es capaz de afrontar, dejando de luchar y ver la realidad con otros ojos, es el momento en que está en condiciones de poder trascenderlo.
La mayoría necesitamos la ayuda y el acompañamiento necesarios para atrevernos a mirar el miedo, la muerte o la amenaza a los ojos y quitarle su poder, el poder que equivocadamente antes se les había dado, y retirárselo y retomarlo para sí mismo.

Un símil del acompañamiento sería reconocer que una de las formas menos amenazantes que tiene un niño de entrar en una habitación oscura es ir de la mano de alguien que no tenga miedo; el acompañamiento de los enfermos se hace desde la confianza, el coraje, la sabiduría y la compasión. Y desde aquí crear el entorno donde el otro no se encuentra solo, ni aislado y si el coraje que percibe el enfermo junto con su intuición de que algo interesante le espera, son superiores a sus miedos y se atreve con cierto respeto a cruzar esta fase para llegar mas allá, entonces puede trascender (atravesar y ascender) y descubrir lo que siempre ha estado allí, a un nivel de conciencia más profundo y más vívido, su propio SER.

Debemos recordar que ante la persona que sufre hay un poder terapéutico de nuestra presencia, entendida como presencia en plenitud.

Como dice Javier Barbero(33), "cuando estamos en medio del sufrimiento, somos co-responsables no solo en eliminar sus causas, sino también en la actitud frente a él. Y, en último término, no será tanto un problema que requiera explicación, cuanto un misterio que demanda presencia". Ver la tabla 3.

El trabajo del profesional en este momento es el de estar presente, desde la serenidad, la confianza y su propia paz interior, viendo y entendiendo el sufrimiento (la amenaza a la integridad) como una forma estrecha e incompleta de verse a sí mismo por parte del enfermo. Desde la visión del terapeuta, que reconoce al mismo tiempo la vulnerabilidad pero también la trascendencia del ser que tiene delante 


\section{Tabla 3. Sufrimiento}

\section{EVALUACIÓN DEL SUFRIMIENTO}

\begin{tabular}{lll}
\hline CATEGORÍA & CONDICIÓN & ESTRATEGIA \\
PROBLEMA & EVITABLE & RESOLVER O PALIAR \\
MISTERIO & INEVITABLE/CONDICIÓN EXISTENCIAL & ACOMPAÑAR \\
\hline
\end{tabular}

sufriendo, se puede, poniendo el espejo de su presencia consciente delante, facilitar que el paciente descubra esta visión más amplia y profunda que la que tiene de sí mismo. Es un momento de intentar recordarle su naturaleza esencial, que pueda verse con los ojos que miran mas allá de la contingencia de la enfermedad y la fragilidad y orientarle en la búsqueda de la dirección adecuada para que él pueda verse con estos nuevos ojos que le permitan trascender la estrecha percepción de sí mismo, causante de su sufrimiento.

El profesional debe ser un acompañante del proceso en esta búsqueda, manteniendo la conexión empática y con su presencia plena creando el espacio de seguridad donde comparte el proceso narrativo del enfermo, mientras mantiene su compasión.

La compasión es, en nuestro entorno cultural, un término bastante desprestigiado por mal comprendido. Es importante entenderlo y diferenciarlo de la lástima. Dice Sogyal Rimponché en el Libro tibetano de la vida y la muerte ${ }^{(34)}$ : "todo lo que se ha dicho sobre la asistencia a los moribundos podría resumirse en dos palabras: amor y compasión. ¿Qué es la compasión? No es solamente una sensación de lástima o interés por la persona que sufre, ni es solamente un afecto sincero hacia la persona que tenemos delante, ni solo un claro reconocimiento de sus necesidades y su dolor; es también la determinación sostenida y práctica de hacer todo lo que sea posible y necesario para aliviar su sufrimiento [...]. La compasión no es auténtica compasión si no es activa."
La compasión a la que nos referimos no es una emoción, sino una actuación a favor del otro para aliviar su padecer. Está excelentemente expresada en la parábola del buen samaritano: " pero el samaritano que iba de viaje, paso por su lado, lo vio y se compadeció, se acercó a él, le puso aceite y vino en las heridas, se las vendó y cuando acabó, lo subió a la montura y lo llevó al hostal y se ocupó de él."

Esta compasión, comienza por la conmoción interna del terapeuta que percibiendo el sufrimiento del otro se siente movido a actuar y transforma la conmoción en un "ocuparse de él", en un actuar, empleando la cabeza, movida por el corazón para ayudar eficazmente a disminuir el dolor y el sufrimiento del otro que es percibido como un igual, como un hermano. Es ofrecer nuestra presencia en plenitud y, desde ella, darle poder para que él se descubra a sí mismo conectado a su propia profundidad.

El mensaje no verbal que el enfermo debe recibir del profesional que le acompaña es: "Tú no estás solo en este proceso y tu idea de estar solo y separado de los demás es un sueño de tu mente, del que debes despertar para encontrar tu integridad como persona". "Estoy aquí para recordarte que somos iguales, que tú eres mucho más de lo que quizás habías sospechado durante toda tu vida y mientras tu cuerpo se deteriora y tus fuerzas físicas disminuyen, vas a ir descubriendo una vida interior, una profundidad en ti mismo, que va a estar cada vez más despierta y te transportará hacia otro espacio de conciencia, Ileno de paz y serenidad." 
Esta compasión es consecuencia de la unión entre el profesional como persona y los demás seres humanos a los que acompaña. $Y$ es posible transmitir confianza y seguridad en este espacio en la medida en que el terapeuta ha visto, ha vivido en algún momento en sí mismo, la experiencia de este espacio de profundidad que se percibe en momentos cumbre. Cuando se para la percepción y se vive sin tiempo, cuando se ha traspasado alguna zona de sufrimiento y se ha comprobado cómo la conciencia continúa y se expande. La compasión no es una actitud psicológica, es más bien fruto de la conciencia madura, un fruto obtenido de la práctica del silencio y de la experiencia de ejercerla.

Eckart Tolle, un maestro espiritual de nuestros días, ha descrito muy bien cómo opera esta compasión, cuando explica ${ }^{(35)}$ : "Hay dos aspectos en la compasión, este aspecto dual es la conciencia de compartir con cada ser humano nuestras dos condiciones, la mortal y la inmortal. En el nivel de la forma, usted comparte la mortalidad y la precariedad de la existencia. En el nivel del Ser usted comparte la vida radiante, eterna. Estos son los dos aspectos de la compasión. En la compasión, los sentimientos aparentemente contrapuestos de tristeza y alegría se mezclan en uno y se transmutan en una profunda paz interior. Esa es la paz de Dios. Es uno de los sentimientos más nobles de los que el ser humano es capaz y tiene un gran poder curativo y transformador".

Un encuentro entre el que sufre y el que le acompaña desde la simetría moral

La aparición de la enfermedad del final de la vida sitúa a la persona ante la necesidad de desbloquear aquellas capas de su self: su estatus, sus roles, las preocupaciones cotidianas intranscendentes, etc. que le han hecho ir perdiendo su identidad más primaria.
El ser humano, entonces, se sitúa desnudo frente a sí mismo y esa desnudez, en principio, provoca temor, incertidumbre y sensación de desprotección. Es ahí donde los recursos espirituales le pueden ayudar a renombrarse a sí mismo, a re-situarse en lo esencial frente a lo accesorio.

Ese proceso, que tiene mucho de regresivo, de vuelta al estado original, no es fácil hacerlo sin ayuda, bien sea de sus propios recursos espirituales y su trabajo interno exploratorio y atrevido, bien sea del apoyo espiritual de su entorno o de los profesionales. Es ahí donde se puede producir el paso del desnudo al vínculo.

Uno puede acabar encontrándose profundamente con uno mismo en la medida en que se vincula con alguien que le sostiene en su experiencia de búsquedasufrimiento. De hecho, parte de nuestro trabajo de apoyo espiritual estriba en la capacidad de sostener en el dolor, durante el viaje que el propio sujeto va haciendo en el interior de sí mismo. Una vez que llega, también paradójicamente, es cuando puede transcender su self y encontrarse con lo que le vincula más allá de su propia existencia.

El problema es que el profesional, para acompañar, también se encuentra desnudo, pues la desnudez humana del paciente le vincula con su desnudez más profunda y eso suele ser temido. En otros ámbitos tenemos herramientas terapéuticas que nos hacen sentirnos seguros: analgésicos para el dolor, ayuda económica y social, cuidados que proporcionan confort...etc. Pero el terreno espiritual es el encuentro persona a persona. Esto no significa una relación privada de amistad, pero sí un encuentro entre la persona-paciente $y$ la persona-profesional, un encuentro por tanto personal. Y aquí es donde nos podemos sentir im-potentes, es decir, sin poder, que es una de las claves habituales que conforma la relación. El encuentro personal-espiritual nos coloca en condiciones 
de simetría moral y nos enfrenta a nuestra propia condición humana. Por eso es tan difícil, por eso estamos ante un reto personal y profesional de hondo calado.

El cultivo de la espiritualidad del

profesional como oportunidad de ayuda y de crecimiento

Aunque el acompañamiento espiritual debería estar disponible para todas las personas que se enfrentan al proceso de morir, no todos los profesionales podrán acompañar. No es algo que se pueda aprender leyendo un manual, está más relacionado con lo que somos que con lo que sabemos y mas con el grado de madurez espiritual que con los conocimientos. Estamos refiriéndonos a la sabiduría del corazón. La capacidad de mantener la actitud compasiva y ecuánime en el entorno del sufrimiento no es innata. Aunque todas las personas tenemos un fondo compasivo y, especialmente los profesionales sanitarios, un corazón compasivo, éste debe cultivarse y equilibrarse con un cierto grado de sabiduría para no verse arrastrado por el dolor del otro.

El profesional que quiera trabajar esta dimensión de la realidad del morir debe proveerse de las herramientas adecuadas, cultivando su propia dimensión espiritual y promoviendo su hospitalidad, presencia y compasión. Afortunadamente hoy disponemos de evidencia de que éstas pueden cultivarse de forma eficiente ${ }^{(36)}$.

En su conferencia plenaria en el congreso europeo (EAPC) de Lisboa en mayo de 2011, Michael Kearney comenzó recordando cómo forjó su vocación de paliativista en el Hospice de St Cristhopher, fundado por Cicely Saunders y que él bautizó como "un lugar de sanación" y acabó diciendo que "la calidad de la presencia que como profesional aportas a la cabecera del enfermo y que puede ayudarle a restablecer sus conexiones sanadoras y trascender el sufrimiento, depende de la calidad de tu vida interior $y$, en este sentido, el autocuidado espiritual del profesional debe verse como un imperativo ético". El acento sobre el "ti mismo" del mensaje de "ama al prójimo como a ti mismo" resume esta necesidad.

La integridad de la persona que tú como terapeuta eres, cuando entra en contacto con el paciente, puede ayudarle a sanar, a trascender el sufrimiento, a facilitarle aceptación, entrega, rendición y entrar en el espacio de trascendencia que describe $\mathrm{K}$ Dowling Singh. Tu presencia, tu madurez espiritual, tu compasión y tu paz interior pueden ayudar a cambiar la experiencia del que tienes delante de ti, seas tú consciente o no de este efecto.

Si estamos de acuerdo con esta visión, debemos entonces aceptar que uno de los aspectos que podemos modificar en el proceso, es el despertar a la dimensión trascendente o espiritual a los profesionales que cuidan pacientes al final de la vida.

Esto implica ayudar a formarse en profundizar en sí mismo para ir perdiendo nuestros miedos, nuestras sombras, ir trascendiendo nuestras cuestiones pendientes y apegos que frecuentemente interfieren en la relación terapéutica. Y desarrollar nuestra capacidad para ser compasivos y ecuánimes en el entorno del sufrimiento. La decisión de hacernos sanitarios suele ser la respuesta a una llamada de nuestro interior, una intuición que nos lleva a sentir que nuestra vocación es la de ayudar a los que sufren y esto es tener un corazón compasivo. Hasta hace poco los profesionales que trabajamos en el entorno del sufrimiento lo hemos hecho intuitivamente, sin formación especifica, sin herramientas y arriesgándonos al contagio emocional, al estrés post traumático secundario a lo que se ha llamado fatiga por compasión o al burnout.

Recientemente hemos comenzado a explorar el curriculum interior de los pro- 
fesionales que trabajan con el sufrimiento y hemos evidenciado la relación significativa y positiva entre el grado de autoconciencia, el autocuidado, la satisfacción de la compasión y la capacidad de afrontamiento ante la muerte. Así mismo la autoconciencia en estos profesionales se relaciona negativamente con la fatiga de compasión y el riesgo de burnout ${ }^{377}$. Sabemos por otra parte que la atención plena, puede cultivarse y desarrollarse e igualmente nuestra capacidad de ser compasivos. Esto abre el camino, ya intuido, para poder cultivar la espiritualidad del profesional y conseguir profesionales más ecuánimes, compasivos y que puedan desarrollar de forma gozosa su trabajo en un entorno para el cual hasta ahora había más riesgos que satisfacción.

Se ha dicho que nadie puede acompañar a otro más allá de donde uno mismo ha llegado y por ello, los que quieran adentrase en el ámbito del acompañamiento espiritual deben dotarse de la consistencia interna, la humildad, la confianza y el coraje que surgen de una mente serena, sabia y compasiva. Hoy sabemos que estas cualidades pueden cultivarse. El reto es crear y difundir las oportunidades para este cultivo.

\section{CONCLUSIONES: LAS CLAVES PARA ACOMPAÑAR}

Cuidar personas- pacientes y sus familiares- en el proceso de morir nos obliga a hacerlo desde un modelo integral que incluya la espiritualidad.

En clínica disponemos de modelos que integran la dimensión espiritual como un universal humano, que entendemos como nuestra naturaleza esencial de donde surge nuestro anhelo inagotable de plenitud. Se expresa a través de la red de relaciones que establecemos, intra inter y transpersonalmente.

El acompañamiento espiritual entendido como la evaluación de los recursos y necesidades de las personas y el acompañamiento de los momentos de máxima vulnerabilidad pueden entenderse como una oportunidad de un cierre armónico de la biografía y una oportunidad de sanación.

Existen modelos y cuestionarios como el del GES que pueden orientar cómo acercarnos a esta dimensión de nuestros pacientes si bien el autentico instrumento de evaluación y acompañamiento es el propio profesional.

El profesional que ha tenido la experiencia personal de afrontar y aceptar su propio sufrimiento o cuando su experiencia de vida le ha llevado a aceptar su mortalidad domesticando el miedo o cuando su compasión hacia los enfermos y sus ganas de aliviar su sufrimiento es mayor que su miedo a morir, entonces puede acercarse a acompañar a los pacientes. Y deberá hacerlo a través de su tristeza y su dolor por tener que morir y una vez atravesado este espeso campo, puede encontrar el gozo de compartir la condición eterna de la dimensión inmortal. En este nivel más profundo, la compasión es sanadora, repara y disuelve el miedo a través de traspasar la visión estrecha y amenazante que nos da nuestra condición mortal.

Se trata de que el profesional, trabaje desde la confianza y el coraje, desde su experiencia de haberse entregado y comprobado que solo gana, aumenta $y$ crece cuando rebaja las expectativas del yo. Desde esta confianza, debe atreverse a acompañar y ayudar al enfermo a recordar que el sufrimiento se encuentra en la superficie de nuestra conciencia y que, en una dimensión más profunda, estamos siempre sanos e íntegros.

Con este modelo en mente, queda evidente que la capacidad de sanar depende también en parte del sanador, de la madurez y experiencia del propio profesional, ya que nadie puede acompañar a otro mas allá de donde uno mismo ha llegado. 
El psicooncólogo tiene herramientas muy útiles -lo da su profesión-, de ámbito comunicativo, para acompañar los procesos espirituales. Ahora bien, esas herramientas son condición necesaria, pero no suficiente para dicha tarea. Eso sí, tiene un ámbito terapéutico privilegiado que facilita el encuadre y la posibilidad de acompañar el viaje. Tiene mucho de responsabilidad, pero también de experiencia apasionante, deseamos que esta revisión pueda ayudar a facilitarla.

\section{REFERENCIAS BIBLIOGRÁFICAS}

1. Sepúlveda C1, Marlin A, Yoshida T, UIIrich A. Palliative Care: the World Health Organization's global perspective. J Pain Symptom Manag 2002;24:91-6. Doi:10.1016/S0885-3924(02)00440-2

2. Casell E. Diagnosing Suffering: A Perspective. Ann Intern Med 1999;131:531-4.

3. Clark D. An annotated bibliography of the publications of Cicely Saunders. Part 1: 1958-67 Pall.Med1998;12:181-93.

4. Clark D. An annotated bibliography of the publications of Cicely Saunders. Part 2: 1968-77 Pall.Med 1999;13:485-501

5. Herdman $\mathrm{TH}$, editor. NANDA Internacional Diagnósticos Enfermeros: Definiciones y Clasificación 2012-2014. Barcelona: Elsevier, 2013.

6. Loevinger J. Ego development. San Francisco: Jossey-Bass, 1977.

7. Kohlberg L. The Philosophy of moral development. Moral stages and the idea of justice. San Francisco, CA: Harper \& Row Pubs, 1981

8. Wilber K. La conciencia sin fronteras. Aproximación de Oriente y Occidente al crecimiento personal. Ed. Kairós Barcelona 1985,p.508-509.

9. Dürckheim KG. Experimentar la trascendencia. Barcelona: Luciérnaga; 1992

10. Grof C, Grof S. La tormentosa búsqueda del Ser: una guía para el crecimiento personal a través de la emergencia espiritual. Barcelona: La liebre de Marzo; 1995.
11. Puchalski C, Ferrell B, Virani R, Otis-Green S, Baird P, Bull J, et al. Improving the Quality of spiritual care as a dimension of paIliative care: The report of the consensus conference. J Palliat Med 2009:12:885-90. Doi: 10.1089/jpm.2009.0142.

12. Nolan S, Saltmarch P, Leget C. Spiritual care in palliative care: working towards an EAPC Task Force. Eur J Pal Care 2011;18:86-9.

13. Puchalski CM,Vitillo R, Hull S.K, Reller N. Improving the spiritual dimension of whole person care: Reaching national and international consensus. J Pall Med 2014;17:115. Doi: 10.1089/jpm.2014.9427.

14. Benito E, Barbero J, Payás A. El acompañamiento espiritual en cuidados paliativos. Una introducción y una propuesta. SECPAL. Madrid: Arán Ed. ; 2008

15. Reed PG. An emerging paradigm for the investigation of spirituality in nursing. Res Nurs Health 1992;15:349e357.

16. Mount BM, Boston PM, Cohen SR. Healing connections: On moving from suffering to a sense of wellbeing. J Pain Symptom Manage 2007;33:372-88. Doi:10.1016/j. jpainsymman.2006.09.014

17. Frankl V. El hombre en busca del sentido último. El análisis existencial y la conciencia espiritual del ser humano. Paidós, 1999.p.189.

18. Sulmasy DP. A biopsychosocial-spiritual model for the care of patients at the end of life. Gerontologist 2002;42:24-33. Doi: 10.1093/geront/42.suppl_3.24

19. Cassell EJ. The person in medicine. Conceptual explorations on person-centered medicine. Int J Integr Care 2010;10:50-2.

20. Egnew T. The meaning of healing: Transcending suffering. Ann Fam Med 2005;3:25562. Doi: 10.1370/afm.313

21. Mount B, Kearney M. Healing and paIliative care: Charting our way forward. Pall Med 2003; 17:657- 8. Doi: 10.1191/0269216303pm848ed

22. Kearney M. Mortally wounded: Stories of soul pain, death and healing. New York: Scribner, 1996. 
23. Mount BM, Boston PH, Cohen SR. Healing connections: on moving from suffering to a sense of well-being. J Pain Symptom Manage 2007;33: 372-388.

24. Mount M. Existential suffering and the determinants of healing. Eur J Pal Care 2003;10:40-2.

25. Reed PG. Spirituality and well-being in terminally ill hospitalized adults. Res Nurs Health 1987;10:335-44.

26. Mako C, Galek K, Poppito SR. Spiritual pain among patients with advanced cancer in palliative care. J Palliat Care 2006;9:1106-13. Doi:10.1089/ jpm.2006.9.1106.

27. Meezenbroek E de J, Garssen B, Van den Berg M, Tuytel G, Van Dierendonck D, Visser A, et al. Measuring spirituality as a universal human experience: Development of the Spiritual Attitude and Involvement List (SAIL). J Psychosoc Oncol 2012;30:141-67. Doi: 10.1080/07347332.2011.651258.

28. Benito E., Oliver A., Galiana L., Barreto P., Pascual A., Gomis C., Barbero J. Development and validation of a new tool for the assessment and spiritual care of palliative care patients. J Pain Symptom Manage 2014;47:1008-18.

29. Singh KD. The grace in dying. How we are transformed spiritually as we die. San Francisco: Harper, 1998.
30. Shaver WA. Suffering and the role of abandonment of self. J Hosp Pall Med 2002:4;46-53.

31. Kubler-Ross E. Sobre la muerte y los moribundos. Alivio del sufrimiento psicológico para los afectados. Barcelona:Ed DebolsiIlo.2003.

32. Nowen H. El Sanador Herido. Madrid: PPC, 1998

33. Barbero J. El apoyo espiritual en cuidados paliativos. Lab Hosp 2002;263:5-24

34. Sogyal Rimpoché. El libro tibetano de la vida y de la muerte. Barcelona: Urano; 1994.

35. Tolle E. Sufrimiento consciente en un mundo nuevo ahora. Barcelona: Grijalbo; 2006.p 96

36. The Center for Compassion And Altruism Research And Education. About Compassion Cultivation Training (CCT). En línea. [Acceso 15 de octubre de 2016]. Disponible en: http://ccare.stanford.edu/ education/about-compassion-cultivationtraining-cct/

37. Sansó N, Galiana L, Oliver A, Pascual A, Sinclair S, Benito E. Palliative care professionals' inner life: exploring the relationships among awareness, self-care and compassion satisfaction and fatigue, burn out, and coping with death, J Pain Sympt Manage 2015:50: 200-7. Doi: 10.1016/j. jpainsymman.2015.02.013 